\title{
La focalización. Génesis y desarrollo de un concepto ${ }^{1}$
}

\section{The focalization. Genesis and development of a concept}

\section{Raquel Gutiérrez ${ }^{2}$ Benemérita Universidad Autónoma de Puebla}

\section{RESUMEN}

Este artículo da seguimiento al desarrollo del concepto de focalización, pues a lo largo de casi ochenta años son notables las diferencias de su planteamiento y comprensión por parte de los distintos autores que lo han abordado. Cuatro ejemplos ilustrarán el uso metodológico del concepto en los trabajos de análisis textual. En tanto recuperación teórica, la noción de focalización se identifica en La República de Platón, en la crítica francesa, en la corriente anglosajona y, finalmente, en la teoría narratológica de Gérard Genette. Si bien el concepto de focalización cambia

${ }^{1}$ Véase (1986, julio-diciembre). Semiosis, (17), pp. 113-135. Xalapa, Universidad Veracruzana. Actualizado.

2 Raquel Graciela Gutiérrez Estupiñán estudió la Licenciatura en Letras Románicas en la Universidad de Lovaina; sus estudios de Maestría los cursó en la Benemérita Universidad Autónoma de Puebla y el Doctorado en filología, en la Universidad Nacional de Educación a Distancia, en Madrid. Actualmente, es profesora-investigadora de posgrado en Ciencias del Lenguaje del Instituto de Ciencias Sociales y Humanidades de la Benemérita Universidad Autónoma de Puebla, donde dirige el Seminario de Análisis cinematográfico. Es miembro del Sistema Nacional de Investigadores del Consejo Nacional de Ciencia y Tecnología. Las líneas de interés académico son la literatura de autoras mexicanas y españolas y el análisis del discurso en textos novohispanos. En el año 2000, obtuvo el Premio nacional de Ensayo "Abigael Bohórquez". Entre sus publicaciones, destacan Una introducción de la teoría literaria feminista (2004) y Once acercamientos al objeto cinematográfico (2015). 
de un autor a otro, es posible afirmar que, en todos los momentos de este estudio, la preocupación por atender la relación narrador / lector / mundo narrado permanece, junto con las maneras de elaborar el mundo de la ficción.

Palabras clave: Focalización; análisis textual; narrador; lector; mundo narrado.

This article follows the development of focalization concept, since over almost eighty years the differences in its approach and understanding by the different authors who have approached to it are notable. Four examples will illustrate the methodological use of the concept in textual analysis works. As a theoretical recovery, the focalization notion is identified in Plato's Republic, in the French Critique, in the Anglo-Saxon current and, finally, in Gérard Genette's narratological theory. Although the focalization concept changes from one author to another, it is possible to affirm that in all moments of this study, the concern to attend the narrator / reader / narrated world relationship remains together with the ways to elaborate the fiction world.

Key words: Focalization; textual analysis; narrator; reader; narrated world.

\section{Introducción}

Dentro de la "exposición de una narratología coherente y sistemática, y de los conceptos que a ella incumben", objetivos de Mieke Bal (1985) en su Teoría de la narrativa, encontré especialmente interesante el apartado que dedica a la focalización. La razón de este interés radica, por una parte, en el desarrollo de la conceptualización técnica de este problema y, por otra, en las posibles aplicaciones para el análisis de textos.

El objetivo de este trabajo es, entonces, estudiar con detenimiento la evolución del concepto de focalización, con el fin de detectar posibles diferencias en el tratamiento del problema por parte de los distintos autores que, a lo largo de casi ochenta años, 
se han interesado en él. Cuatro ejemplos ilustrarán las aplicaciones que se han hecho del concepto de focalización al análisis textual en diferentes etapas de la constitución de la teoría que nos ocupa.

\section{Point of view}

En La República, Platón establecía ya una distinción entre "narración simple" -si el poeta aparecía en todas partes de la narración, sin esconderse- e "imitación" - si el poeta asimilaba su estilo al de otra persona, por la voz o por el gesto. Para ilustrar esta diferencia, Platón trasladó un fragmento de la Ilíada del estilo directo al indirecto, es decir, cambiando el fragmento de imitativo a narrativo.

Sin embargo, el desarrollo de la aplicación específica de esta distinción básica al análisis del punto de vista arranca de las reflexiones que James (1934) expone en sus prefacios. Un problema que le obsesionaba era el de encontrar un "centro", un "focus" narrativo para sus obras, problema que, pensaba, podía resolverse limitando la materia narrativa al marco de la conciencia de uno de los personajes de la trama. Escribía: "There is no economy of treatment without an adopted, a related point of view...” (pp. 37-38).

Según Zéraffa (1971), James no cesó de perfeccionar "una técnica de focalización interna de la obra", pero Zéraffa advierte que sería un error confundir "focus" con "punto de vista" porque el universo de las novelas de James no es aprehendido en el haz luminoso de la individualidad pura: entre el personaje y su entorno, el escritor establece un sistema constante de intercambios e interferencias. La novela está focalizada según la mirada privilegiada de un personaje localizado (1971, p. 60).

La influencia de James es clara en los autores que después de él se ocuparon del mismo problema. Lubbock (1921) definió el punto de vista como el problema de la relación que existe entre el narrador y el relato y distinguió dos métodos para representar el mundo ficticio: panorámico - palabra y gesto referidos por el narrador- y escénico -palabras y gestos "dramatizados" directamente. Pero cuando escribe "The art of fiction does not begin until the novelist thinks of 
his story as a matter to be shown, to be so exhibited that it will tell itself rather than being told by the author [...] the thing has to look true, and that is all. It is not made to look true by simple statement" (p. 62), lo que hace es suscitar dudas, más que dar respuestas.

El desarrollo inminente del concepto de punto de vista no dejó de provocar polémicas. Mientras Forster (1927) ve el problema como "a trivial technicality" (p. 118), Allen Tate (1941) y Phyllis Benthey (1947) se pronuncian por la "autoridad de la acción".

Por su parte, Mark Schorer (1948) da un paso adelante con respecto a Lubbock al examinar los usos del punto de vista no sólo como un modo de delimitación dramática, sino, más particularmente, de definición temática.

Muy interesantes son las observaciones de Brooks y Warren (1947), por cuanto revelan una mayor formalización y porque se perfila ya una distinción entre "focus of narration" y "point of view". Estos autores plantean el problema del foco de la narración en términos de ¿quién ve? o ¿quién cuenta?, y distinguen cuatro tipos de "focus", que ilustra el esquema siguiente:

Tabla 1:

\begin{tabular}{|l|l|l|}
\hline & $\begin{array}{l}\text { Internal analysis of } \\
\text { events }\end{array}$ & $\begin{array}{l}\text { Outside observation of } \\
\text { events }\end{array}$ \\
\hline $\begin{array}{l}\text { Narrator as a charac- } \\
\text { ter in the story }\end{array}$ & $\begin{array}{l}\text { (1) Main character } \\
\text { tells his story }\end{array}$ & $\begin{array}{l}\text { (2) Minor character tells } \\
\text { main character's story }\end{array}$ \\
\hline $\begin{array}{l}\text { Narrator not a charac- } \\
\text { ter in the story }\end{array}$ & $\begin{array}{l}\text { (4) Analytic of omnis- } \\
\text { cient author tells story }\end{array}$ & $\begin{array}{l}\text { (3) Author tells story as } \\
\text { observer }\end{array}$ \\
\hline
\end{tabular}

Brooks y Warren (1947, p. 589),

es decir, (1) un personaje cuenta su historia en primera persona: narración en primera persona; (2) un personaje puede contar, en primera persona, algo que ha observado: observador en primera persona; (3) el autor puede contar lo que pasa en sentido puramente objetivo -hechos, palabras, ademanes-, sin penetrar en la mente de los personajes y sin dar su propia opinión: autor-observador; (4) el autor puede contar lo que pasa con plena libertad de penetrar en 
la mente de los personajes y de dar su propia opinión: autor omnisciente. Hay posibilidad de combinar los métodos.

Al referirse al término "punto de vista”, Brooks y Warren (1947) hacen notar que puede usarse en dos diferentes acepciones: a) con respecto a la actitud básica o idea del autor -en esta acepción, se hace referencia, por ejemplo, al punto de vista "irónico" o inclusive "cristiano" del autor; b) con respecto al modo de la narración -en este caso, se hace referencia a la mente a través de la cual se presenta el material de la narración: primera persona, autor-observador, autor omnisciente, como vimos antes.

Para evitar confusiones, proponen restringir el uso del término "punto de vista" para la primera acepción y emplear el de "foco de la narración" -focus of narration- para la segunda.

El problema del narrador para Norman Friedman (1955) consiste en la adecuada transmisión de su narración al lector. En un artículo titulado "Point of view in fiction: the development of a critical concept", este autor, además de hacer una síntesis de los diferentes estudios hechos hasta el momento en Estados Unidos e Inglaterra sobre el punto de vista, propone una tipología de los modos de transmisión del material narrado. Como base de esa tipología establece como distinción principal aquella que existe entre "summary narrative" (decir) e "immediate scene" (hacer). La primera consiste en un relato generalizado de hechos que abarcan un determinado periodo y una variedad de lugares; la segunda se da cuando surgen detalles específicos y sucesivos de tiempo, lugar, acción, diálogo. Por supuesto, estos dos modos no se dan en estado puro. Examinemos brevemente la tipología que Friedman propone.

Omnisciencia editorial (editorial omniscience). El término omnisciencia significa un punto de vista ilimitado -y por ello, difícil de controlar. La marca característica de este tipo de enfoque son las intrusiones del autor; en consecuencia, éste no sólo cuenta lo que sucede en la mente de sus personajes, sino que también lo critica.

Omnisciencia neutral (neutral omniscience). Este segundo paso hacia la objetivación difiere del primero por la ausencia de intrusiones del autor, que habla impersonalmente en tercera persona. 
Con respecto a estos dos tipos en que aparece la omnisciencia, Friedman insiste en que la característica dominante es que el autor siempre está dispuesto a intervenir entre el lector y la narración, y que aun cuando disponga una escena, la contará como él la ve y no como sus personajes podrían verla.

"Yo" como testigo ("I" as witness). Al delegar el autor sus "poderes" sobre otro, la consecuencia es que en el marco narrativo de este tercer tipo ya no le es posible el acceso a los estados mentales de los personajes. Sin embargo, lo que el testigo transmite al lector puede enriquecerse a través de sus contactos con los otros personajes y de las inferencias acerca de sus sentimientos o pensamientos.

"Yo" como protagonista ("T" as protagonist). Aquí uno de los personajes -generalmente uno de los principales- cuenta su propia historia, en primera persona. Este protagonista-narrador tiene una gran movilidad y acceso a más información que el mismo protagonista, envuelto en la acción; está casi enteramente limitado a sus propios pensamientos y percepciones.

Omnisciencia selectiva múltiple (Multiple selective omniscience). La adopción de este modo por parte de la voz narrativa implica la eliminación tanto del autor como de cualquier narrador posible. La apariencia de los personajes, lo que hacen o dicen, sólo puede ser transmitido al lector a través de la mente de alguien presente. ${ }^{3} \mathrm{La}$ diferencia entre este tipo de omnisciencia y la omnisciencia "normal" es que mientras la primera presenta los sentimientos, percepciones y pensamientos como ocurren consecutivamente y en detalle a través de la mente (escena), la segunda presenta un resumen de ellos y los explica después que han ocurrido (narración).

Omnisciencia selectiva (selective omniscience). El lector está limitado a la mente de uno solo de los personajes; en lugar de disponer de una variedad de ángulos, se encuentra fijo en el centro.

${ }^{3}$ Véase como ejemplo de esta técnica la novela de Virginia Woolf, To the Lighthouse: "They must find a way out of it all. There might be some simpler way, she sighed. When she looked in the glass and saw her hair grey, her cheek sunk, at fifty, she thought, possibly she might have managed things better her husband; money his book." 
Modo dramático (the dramatic mode). La información de que el lector dispone se reduce a lo que los personajes hacen y dicen; no hay ninguna indicación acerca de lo que ven, piensan o sienten. Hay una notable analogía con el drama, en el sentido de que el lector no "escucha" más que a los personajes, mismos que se mueven como en un escenario; su ángulo de visión es fijo y la distancia siempre es corta.

La cámara (the camera). La reseña de posibles puntos de vista concluye con el último extremo de la exclusión del autor. Se trata de transmitir, sin selección ni arreglo aparentes, "une tranche de vie", ${ }^{4}$ como si pasara ante una cámara (Friedman, 1955, 1179).

La influencia de James (1934) es aún muy fuerte en Friedman (1955). Afirma este autor: "The choice of a point of view in the writing of fiction is at least as crucial as the choice of a verse form in the composing of a poem... (p. 1180). Y así como bay cosas que no pueden decirse en un soneto, a cada una de las categorias que él propone le concierne un tipo de funciones que puede contener dentro de sus límites. Entonces, la elección de una u otra categoría dependerá de lo que el autor-narrador quiera presentar.

La principal contribución de Wayne C. Booth (1961) parece ser la introducción de la noción del "reliable" o "unrealiable" narrator, así como la del autor implícito. Antes de examinar estos conceptos, hagamos notar que sus propuestas constituyen un paso adelante con respecto a los autores de los que hemos hablado antes. En efecto, mientras éstos se centran en la relación narrador/historia y en la creación de tipologías, a Booth le preocupa la manera en que el autor puede entrar en contacto con el lector y proporcionarle cierta visión del mundo. Además, mientras en autores anteriores -y aun en más modernos, como luego se verá- hay una confusión constante entre autor y narrador, en Booth aparece ya un intento por llegar a una diferenciación de ambos conceptos.

Para empezar, Booth llama la atención acerca de las intrincadas relaciones entre el llamado autor real y las diversas versiones oficiales de sí mismo: "We must say various versions, for regardless

\footnotetext{
4 "Un trozo de vida".
} 
of how sincere an author may try to be, his different works will imply different versions, different ideal combinations of norms" (1961, p. 71). Esta "versión oficial del autor" es como un "segundo sí mismo" para el que, dice Booth, no hay un término totalmente adecuado: "persona", "máscara", "narrador". Este último es, generalmente, usado para significar el "yo" de una obra, pero el "yo" es rara vez -o nunca- idéntico a la imagen implícita del artista. En otro lugar de su libro, vuelve a aparecer esta distinción cuando, al hablar de las variaciones de distancia, dice que el narrador puede estar 1) más o menos distante del autor implícito -la distancia puede ser moral, intelectual o "It may be physical or temporal: most authors are distant from even the most knowing narrator in that they presumably know "everything turns out in the end"' (pp. 155156)-; 2) más o menos distante de los personajes de la historia que cuenta; 3) más o menos distante de las normas del lector.

En cuanto al autor implícito -the author's "second self"-, afirma Booth que aun las novelas sin narrador dramatizado crean el retrato implícito de un autor, que permanece detrás de la escena, ya sea como director, como titiritero o como un dios indiferente. Este autor implícito es siempre diferente del autor real -"real man"-, que crea una versión superior de sí mismo al crear su obra. Como en el caso del narrador, el autor implícito puede estar más o menos lejos del autor o de los personajes.

Aunque la terminología no le satisface por completo -"for lack of better terme"-, Booth llama "reliable narrator" a aquel que habla o actúa de acuerdo con las normas de la obra -es decir, las normas del autor implícito-; el "unreliable narrator" es el que habla o actúa en contra de esas normas.

Por lo que respecta al problema específico del punto de vista, Booth considera que la clasificación tradicional en tres o cuatro categorías, variables sólo por la persona y el grado de omnisciencia, es inadecuada, porque términos como "primera persona" u "omnisciente" no nos dicen nada acerca de la diferencia entre uno y otro. Tomando en cuenta que la narración es un arte y no una ciencia, surge la pregunta sobre dónde encontrar los principios generales. Para el novelista, las clasificaciones de la crítica no son nin- 
guna ayuda. Frente al problema del punto de vista, el novelista está ante el trabajo individual. Para Booth, como para James, la mayoría de las elecciones son elecciones de grado, no de tipo. El problema no es decidir si el narrador va a ser omnisciente o no, sino, según Booth, "just how inconscient shall he be?" (p. 165).

Concluye esta parte de su exposición afirmando que, en lugar de las reglas abstractas sobre consistencia y objetividad en el uso del punto de vista, lo que se necesita son descripciones específicas acerca de cómo están narradas las grandes obras.

En su artículo acerca de la focalización, Patricia Rubio (1981) menciona a Stanzel y Romberg como los autores más relevantes que, dentro de la crítica alemana, se han ocupado del problema de la perspectiva adoptada por el narrador. Sus propuestas parecen situarlos dentro del tipo de estudios que hemos examinado hasta ahora, puesto que se refieren al establecimiento de tipologías. Stanzel establece tres situaciones narrativas típicas -autorial, en primera persona y situación narrativa personal- y Romberg cuatro tipos de relato -objetivo, en primera persona, con autor omnisciente y con punto de vista.

A pesar de que la propuesta de Booth constituye un enfoque diferente con respecto a los demás autores que se han ocupado del problema del punto de vista, es necesario reconocer que, como pertenecientes a la crítica literaria tradicional, todas las propuestas están centradas en las narraciones narrador/historia y en todas hay una mayor o menor confusión entre autor y narrador. Esta confusión persiste en autores más recientes, pero cultivadores del mismo tipo de crítica literaria. Como ejemplo, citaremos a Zéraffa (1971), quien partiendo de las mismas ideas de James sobre el punto de vista, sostiene que "le romancier a toujours un regard souverain sur son objet, dans les limites qu'il doit nécessairement asigner à ce regard. De plus, à l'in térieur de ces mêmes limites, le romancier sait tout de cet objet” (p. 36). Y Zéraffa realiza sus análisis -por lo demás, muy brillantes- basado en esta idea. 


\section{La crítica francesa}

Cuando pasamos a examinar las actitudes de la crítica francesa con respecto al punto de vista, nos encontramos con que no hay estudios sistemáticos sobre ese problema. Esto se debe, tal vez, a que los franceses se preocupaban sobre todo por la génesis de las obras y por problemas filosóficos, morales o psicológicos. No obstante, podríamos señalar como antecedentes de los estudios más recientes, de Todorov $(1967,1971)$, Genette $(1966,1969,1972)$ y Bal (1985), algunos textos aislados en Sartre (1947) y Prévost (1942), los artículos de Laffay y Doniol-Valcroze, la obra de E. Magny (1948) y la tesis de Georges Blin sobre Stendhal (1954).

La propuesta de Jean Pouillon (1946) es interesante por su influencia sobre Todorov y Genette. Pouillon (1970) no creía que las "visiones" del relato fueran una cuestión técnica y su concepción es psicologista, es decir, para él las visiones del relato están estrechamente relacionadas con la psicología de los personajes. Estas "visiones" son agrupadas por Pouillon en tres tipos básicos, dependiendo del grado de conocimiento que el narrador tiene del mundo y los personajes: a) visión "con", caracterizada por la elección de un solo personaje, que será el centro de la narración y a partir del cual "vemos a los otros"; b) visión "por detrás", en la que el autor, en vez de situarse en el interior de su personaje, intenta "separarse de él, no para verlo desde fuera [...], sino para considerar su vida psíquica objetiva y directamente" (pp. 60-70) -vemos que la diferencia entre uno y otro tipo de visión se reduce a la que existe entre la pura y simple conciencia y el conocimiento reflexivo-; c) la visión "por fuera" se interesa por la conducta, por la apariencia física y por el ambiente sólo en tanto que son datos reveladores de un "dentro", es decir, una psicología.

De esta clasificación, un tanto restrictiva, Genette retendrá -como veremos más adelante- sólo la idea capital: el narrador está dentro o fuera de la historia que cuenta.

Las aportaciones de Todorov con respecto a la teoría sobre el punto de vista son doblemente importantes. Por un lado, representan una especie de enlace entre la concepción anglosajona y la fran- 
cesa, en el sentido de que se apartan tanto de la concepción psicologizante de Pouillon como de las ideas de Lubbock, Friedman y Booth. Con respecto a estos últimos, la renovación de Todorov consiste en haber separado las visiones a aspectos de los "registros del habla", es decir, diversos tipos de discursos narrativos utilizados en el relato, cuestiones que los autores americanos concebían como un único aspecto. Todorov (1971) define la visión como "la manera en que los acontecimientos relatados son percibidos por el narrador y, en consecuencia, por el lector virtual" (p. 120). Todorov establece una distinción entre representación del narrador y no representación del narrador, según que el relato represente o no su propio proceso de enunciación. Dentro de esta división primordial, postula diversas dimensiones significativas, que definen cada tipo particular de visión. Con respecto a la relación entre el narrador y los personajes, Todorov recupera la distinción de Pouillon entre visión "desde dentro" -en la que el personaje no tiene secretos para el narrador- y visión "desde afuera" -en la que el narrador puede describir los actos del personaje, pero ignora sus pensamientos-, ampliando considerablemente esos conceptos. ${ }^{5}$

Y así llegamos a Gérard Genette, cuya tipología es la actualmente "vigente". Antes de examinar sus propuestas, podemos establecer una distinción entre la actitud de la crítica a partir de Pouillon y los autores de la corriente anglosajona.

Me parece que se puede ver claramente que en los autores de la corriente francesa ya no está presente la preocupación por distinguir -O, más bien, la tendencia a confundir- autor/narrador, sino que parten directamente del narrador, dando por supuesta su diferencia con el autor y dejando a éste fuera del análisis. Esta tendencia es la que va a prevalecer en los últimos estudios de narratología.

${ }^{5}$ En el Diccionario enciclopédico de las ciencias del lenguaje, el término de visión o punto de vista se define como "la relación entre el narrador y el universo representado" (Ducrot y Todorov, 1972, p. 369). 


\section{La focalización}

El último paso, hasta ahora, hacia el establecimiento del concepto de focalización lo ha dado Gérard Genette (1972). Al hacer un inventario de los estudios teóricos sobre el "punto de vista", señala que todos los ensayos de designación adolecen de la confusión entre lo que él llama modo, es decir, el punto de vista propiamente dicho -¿quién ve?- y la voz, o sea, la identidad del narrador o de la enunciación narrativa -¿quién habla?-, lo que se constituye en dos series de problemas que debieran ser objeto de una clasificación aparte. Después de hacer esta distinción, Genette establece una tipología de la focalización, en la que se nota la influencia de Brooks y Warren, por un lado, y la de Pouillon, por otro. Los tipos de focalización que Genette distingue son los siguientes.

Focalización cero o ausencia de focalización. El objeto es conocido desde todos los ángulos y desde ninguno. En consecuencia, el narrador entrega más información de la que los personajes poseen.

Focalización externa. El foco se define como exterior al objeto. El conocimiento se detiene en las apariencias, conforme a las condiciones de la percepción humana. La mirada, plenamente objetiva, no es la de nadie.

Focalización interna. El foco está situado en la conciencia de un sujeto-testigo. Para Genette, esta es la única focalización real. El narrador se limita a referir la información percibida por el personaje focalizador y son posibles tres modalidades: 1) focalización interna fija -un solo personaje realiza el proceso de focalización-; 2) focalización interna variable -varios personajes intervienen-; 3) focalización interna múltiple -un mismo hecho es presentado desde distintos ángulos.

En el apartado que dedica a la focalización, Mieke Bal (1985) hace notar que focalización es un "nuevo término para un concepto parcialmente antiguo". Con el término focalización, se refiere a las relaciones entre los elementos presentados y la concepción a través de la cual se presentan. La focalización será, por lo tanto, la relación entre la visión y lo que se "ve", lo que se percibe (p. 108). 
Al observar que las diversas tipologías sobre el punto de vista han confundido al agente que ve con el agente que narra y al distinguir dos tipos de focalización -interna y externa-, ${ }^{6}$ Bal no hace más que confirmar lo que ya había sido establecido por Genette. La utilización de una serie de términos específicos -sujeto focalizador/ objeto focalizado, señal de acoplamiento, objeto de focalización perceptible/no perceptible, bisagra- revela un mayor refinamiento en el manejo de la focalización como herramienta para el análisis.

Bal distingue varios niveles de focalización. En un primer nivel, la focalización es externa. El focalizador está ubicado en un nivel externo a la diégesis. En un segundo nivel, el focalizador es interno. El sujeto es figura de la diégesis. Afirma Bal que "son posibles más niveles", pero no da ejemplo de ello.

Señala Patricia Rubio otras dos contribuciones de Bal, que implican modificaciones al modelo de Genette: 1) la afirmación de que todo relato tiene una instancia focalizadora -para Genette, un texto está focalizado sólo si el proceso se realiza a partir del personaje-, con lo que el concepto de focalización se amplía; 2) la segunda contribución se refiere a que, en lugar de tener una reducción de foco entre la focalización cero e interna y externa, se trataría de funciones diferentes.

Veamos ahora un resumen de los tipos de focalización propuestos a partir de Pouillon. Se trata de un cuadro elaborado por B. Valette (1985), y en el que hemos incluido las contribuciones de Bal.

${ }^{6}$ Aunque en otro lugar del mismo apartado menciona la posibilidad de que el focalizador externo observe junto a una persona, lo que la aproximaría a la "vision avec" de Pouillon. 
Tabla 2:

\begin{tabular}{|c|l|}
\hline \multicolumn{2}{|c|}{ CUADRO DE LOS TRES TIPOS DE FOCALIZACIÓN } \\
\hline I. & Visión "por detrás" (Pouillon) \\
& Narrador> personaje (Todorov) \\
& Focalización cero (Genette) \\
& Mirada de Dios (Valette) \\
& Focalizador extradiegético \\
& Primer nivel de focalización (Bal) \\
\hline II. & Visión "con" \\
& Narrador = personaje (Pouillon) \\
& Focalización interna (Todorov) \\
& Conciencia de un sujeto-testigo (Genette, Patillon) \\
& Focalizador intradiegético (Bal) \\
Segundo nivel de focalización (Bal)
\end{tabular}

Pouillon, 1970; elaboración propia

\section{Aplicaciones del concepto de focalización al análisis de textos}

Un instrumento de análisis sólo se revela eficaz si puede probar su aplicabilidad inmediata. Por esto, nos ha parecido indispensable presentar algunos ejemplos de cómo diversos autores han aplicado el concepto de focalización al estudio de textos específicos. Dado que nuestro trabajo pretende presentar un panorama general de la evolución del concepto de punto de vista, empezaremos por mostrar cómo Brooks y Warren analizan el uso del observador de primera persona en "A Rose for Emily", de Faulkner. Dando un 
salto en el tiempo, daremos un ejemplo de análisis realizado por Gérard Genette. Hablaremos brevemente del análisis que Patricia Rubio hace de los cuentos de Onelio Jorge Cardoso. Y finalmente, presentaremos dos ejemplos tomados de M. Patillon.

¿Cuál es la lógica, preguntan Brooks y Warren, del uso del observador de primera persona en "A Rose for Emily"? El empleo de uno de los habitantes del pueblo para presentar la historia permite al autor indicar, constantemente y a través de mil maneras -metáforas, comentarios, etc.-, la actitud del pueblo hacia Emily y de ella hacia el pueblo. Si se hubiera usado un foco ${ }^{7}$ de tercera persona, esta relación podría especificarse y analizarse, pero no habría sido tan persuasiva, no habría entrado en forma tan completa en la textura de la narración. Además, el hecho de que el narrador está limitado en sus oportunidades de observación, de que sólo ve a Emily por momentos, proporciona un marco altamente dramático para el relato, un artificio para el suspenso. La creciente curiosidad en el pueblo proporciona el modelo que el lector va a seguir en su propio esfuerzo para acercarse a Emily, para comprenderla a ella y el significado de su acción ${ }^{8}$ (p. 591).

Se lee en Gustave Flaubert (1857):

Et aussitôt, reprenant sa course, elle passa par Saint-Sever, par le qua des Curandiers, par le quai aus Meules, encore une fois par le pont, par le place du Champ de Mars et derrière les jardins de l'hospital, où des vieillards en veste noire se promènent au soleil, le long d'une terrasse toute verdie par des lierres.

Este fragmento, citado por Genette (1966), es comentado así por el autor:

7 Genette acuñaría más tarde el término "focalización” a partir de este empleo en Brooks y Warren, que, a su vez, es una clara metáfora fotográfica.

${ }^{8}$ El contenido de este último apartado es una traducción mía. 
Puede uno imaginarse fácilmente que, a esa velocidad y en esas circunstancias, ni Emma ni León tienen la ocasión de contemplar una terraza cubierta de hiedra, y de todas maneras las persianas (de la carroza) están bajadas. Su infortunado cochero, agotado y muerto de sed, tiene otras preocupaciones. Así, desde el punto de vista de las reglas de las narraciones realistas, esta descripción, aunque muy breve, pero aquí también indefinidamente prolongada por su verbo en presente, está muy poco en situación, y muy mal justificada dramática y psicológicamente. Ese primer plano inmóvil en medio de una carrera desenfrenada es la torpeza misma. En realidad un descuido como éste sólo puede significar que la "besuqueada" ambulante no le interesa mucho a Flaubert, y de pronto, al pasear por el jardín del hospital, se pone a pensar en otra cosa. ${ }^{10}$

Patricia Rubio (1981) hace algunas modificaciones a la tipología de las focalizaciones, adoptando las modalidades de Genette y los niveles de focalización de Bal. Es un ejemplo muy ilustrativo de cómo los conceptos teóricos deben afinarse y sufrir ajustes al aplicarlos al análisis de textos, enriqueciendo así la teoría. Citaremos únicamente tres ejemplos de focalización en narraciones heterodiegéticas.

1. Focalización cero ${ }^{11}$ (efectuada por un focalizador no-personal, externo a la diégesis).

\section{Primer nivel de focalización.}

Con los años había crecido también el hijo del policía. Pero el resto de los muchachos prefería no meterse en juegos con aquel muchacho que aprendía las cosas difícilmente y que despertaba a todos un agudo deseo de burla ("Moñigueso").

${ }^{9}$ Recordemos que, en este momento de la novela, Emma ha ido a buscar a León a Rouen y, después de visitar la catedral, inician su relación amorosa... dentro del coche, precisamente.

${ }^{10}$ El contenido de este último apartado es traducción mía.

${ }_{11}$ Patricia Rubio utiliza este término por razones de claridad únicamente, puesto que considera, siguiendo a Bal, que en todo relato hay focalización. 


\section{Focalización interna.}

Segundo nivel de focalización.

Nivel focalizador intradiegético

Segundo nivel

(Eduwiges es figura de la diégesis)

En principio Eduwiges no pensó en la mujer de Trinidad, sino en ella, en Memé, parada sola frente a la puerta, sin la ayuda de nadie, sin el sillón detrás ni delante, ni al lado, sola sin el sillón, sola en vida caminando... ("Memé”)

Nivel narrativo

(Narrador extradiegético)

Primer nivel

3. Focalización doble.

Objeto

focalizado (la cintura, las manos)

Entonces el viejo le recorrió la cintura con los ojos y acabó mirándole las manos ("Camino de las lomas").

Aquí tenemos un sujeto focalizador extradiegético, ejerciendo una focalización interna -primer nivel-y, además, un focalizador del personaje -segundo nivel. El viejo es objeto focalizado del primer nivel de focalización y sujeto focalizador del segundo nivel.

Por último, veamos dos ejemplos de análisis presentados por Michel Patillon (1974). ${ }^{12}$

A. Tout à coup on partit au grand galop. Quelques instants après, Fabrice vit, [à vingt pas en avant, une terre labourée qui était remuèe d'une façon

${ }^{12}$ Los fragmentos citados por Patillon se presentan tal como aparecen en su libro. He traducido, en cambio, el texto del análisis. 
singulière. Le fond des sillons était plein d'eau, et la terre fort humide, qui formait la crète de ces sillons, volait en petits fragments noirs lancès à trois ou quatre pieds de haut.] Fabrice remarqua en remit à songer a la gloire du meréchal. Il entendit [un cri sec auprès de lui]: c'etaient deux hussards qui tombaient atteints par des boulets; et, lorsqu'il les regarde [ils étaient déjà à vingt pas de l'escorte). Ce qui lui sembla horrible, ce fut un cheval tout sanglant qui se débattait sur la terre labourée, en engageant ses pieds dans ses propres entrailles; il voulait suivre les autres: le sang coulait das la boue.

Ah! m’y voild donc enfin au feu ! se dit-il. J'ai vu le feu! se répétait-il avec satisfaction. Me voici un vrai militaire. A ce moment, l'escorte allait ventre à terre, et notre héros comprit que c'étaient des boulets qui faisaient voler la terre de toutes parts. Il avait beau regarder du côte d'ou venaient les boulets, il voyait [la fumée blanche de la batterie à une distance énorme], et, [au milieu du ronflement égal et continu produit par les coups de canon], il lui semblait entendre [des décharges beaucoup plus voisines]; il n'y comprenait rien du tout (Stendhal, 1840, III).

El texto funciona primero en focalización cero, aun cuando se proponga seguir siempre al personaje del héroe: Fabrice. Pero los fragmentos entre corchetes están en focalización interna: el objeto es, entonces, conocido desde el punto de vista de Fabrice. Las expresiones en caracteres más gruesos introducen esta focalización con ayuda de verbos de conocimiento, de la misma manera en que un enunciado cita un discurso indirecto con ayuda de verbos de pensamiento o de elocución. [...]. No hay nada que nos permita decir si detalles como "Le fond des sillons était plein d'eau, et la terre for humide, qui formait la crête de ces sillons, volait en petits fragments noirs lancés à trois ou quatre pieds de haut" o bien "ils étaient déjà à vingt pas de l'escorte" no están en focalización cero. Lo que sí es seguro es que el texto se prohíbe presentar más de lo que Fabrice puede saber. Para el último caso, el texto da cuenta del desplazamiento del foco interno:

Il entendit un cri sec auprès de lui; c'étaient deux hussards qui tombaient atteints par des boulets; et, lorsqu'il les regarda, ils étaient déjà à vingt pas de l'escorte/Tout à coup elle se souvint de l'homme écrasé le jour de sa première recontre avec Vronksi,/ et elle comprit ce qu'il lui restait à faire. /D’un pas rapide et léger elle descendit les marches/et, postée près de la 
voie, elle scruta [les oeuvres basses du train qui la frôlait, les chaines, les essieus, les grandes rues de fonte, cherchant à mesurer de l'oeil la distance qui séparait les roues de devant de celles de derrière]/<"Lá>, se dit-elle en fixant [dans ce trou noir les traverses recouvertes de sable et de pous/siere]< lá au beau milieu; il sera pui et je serai délivrée de tout et dé moi-même”>/

Son petit sac rouge/ qu'elle eut quelque peine a détacher de son bras,/ lui fit manuer le moment de se jeter sous le premier wagon:/ force lui fut d'anttendre le second./ Un sentiment semblable à celui qu'elle éprouvait jadis avant de faire un plongeon dans la rivière s'empara d'elle,/ et elle fit le signe de la croix./ Ce geste familier rèveilla dans son âme une foule de souvenirs d'enfance et de jeunesse; / les minutes heureuses de sa vie scintillèrent un instant à travers les ténèbres qui l'enveloppaient. Cependant elle ne quittait pas des yeux [le wagon],/ et lorsque [le milieu entre les deux roues] apparut,/elle rejeta son sac, /rentra sa tête dans les épaules/ et, les mains en avant, se jeta sur les genoux sous le wagon, comme prête à se relever. /Elle eut le temps d'avoir peur./<"Où suis-je? Que fais-je? Pourquoi?” pensa-t-elle, faisant effort pour se rejeter en arrière. /Mais une masse enorme, inflexible, la frappa à la tête / et l'entraina par le dos. / "Signeur, pardonnez-moi"> murmura-t-elle, sentat L'inutilité de la lutte. Un petit homme, marmottant dans sa barbe, tapotait le fer au-dessus d'elle. /Et la lumière qui pour l'infortunée avait èclairé le livre de la vie, avec ses tourments, ses trahisons et ses douleurs, brilla soundain d'un plus vif éclat,/illumina les pages demeurées jusqu'alors dans l'ombre, /puis crépita, /vacilla, /et, s'éignit pour toujours" (Tolstoi, 1878, vII, p. 21).

El relato del suicidio de Anna Karenina está en el modo de enunciación histórica ${ }^{13}$ y en focalización cero: el narrador conoce todo el acontecimiento; da cuenta de los pensamientos y de las visiones de la heroína, inclusive de los últimos momentos de conciencia durante los cuales, según se cree, la vida transcurrida vuelve a pasar en la memoria. Manifiesta cierta simpatía hacia la heroína, como cuando dice "la infortunada", pero su mirada es ante todo la de un observador cuidadoso.

Los elementos de este fragmento:

$<>$ "relais de parole" (relevo de palabra)

${ }^{13}$ Para la distinción entre récit historique/ discours, véase Benveniste (1966). 


\section{[ ] focos \\ a efectos de realidad \\ // estructura narrativa}

contribuyen, en conjunto, a crear el efecto sobrecogedor. En los relevos de palabra, la palabra con-por la repetición, la interrogación, la exclamación - y la visión con -por la presencia del objeto, las cadenas, los ejes, las enormes ruedas de acero, hasta la arena y el polvo que cubren los durmientes en el hoyo negro- son notables por su intensidad. Pero esos discursos directos y esas visiones en focalización interna no son más que el afloramiento [...] de un acontecimiento restituido con fuerza por el resto del enunciado. Lo mismo para los efectos de realidad. Los enunciados en caracteres gruesos sobre la bolsa roja y el hombrecillo que tamborilea y habla entre dientes -cuatro segmentos narrativos y uno descriptivo- parecen esenciales con respecto a la acción central, es decir, la misma presencia de los objetos, notable en focalización interna, se vuelve a encontrar en focalización cero. Podemos ampliar la observación y considerar que la narración se obliga a presentar únicamente el acontecimiento que se supone ha vivido el personaje central. De ahí el silencio sobre todo lo demás, en particular sobre los testigos de la escena. Además, al condensar el relato sobre un rasgo no-esencial, se deforman las proporciones normales y esta es una manera de hacer ver el objeto, de imponer su presencia [...]. Entonces, en donde el funcionamiento de este texto se revela mejor es en la continuidad que une la parte del texto en enunciación histórica y en focalización cero con la parte que está en "relais".

\section{Conclusiones}

En conjunto, podemos observar una tendencia bien definida hacia un análisis en el que todo queda dentro del relato y en el que el autor acaba por convertirse en un problema aparte. La confusión entre autor y narrador, de la que hablamos al finalizar el punto 2, prácticamente desaparece a partir de Pouillon y sobre todo a partir de Todorov. 
A pesar del distinto desarrollo del problema del punto de vista en la corriente anglosajona y en la francesa, puede hablarse de continuidad: el concepto se va afinando a medida que los diferentes autores lo estudian y se va enriqueciendo con las modificaciones que cada uno hace. Así, vemos que, donde Lubbock, Friedman y Booth habían visto un solo aspecto, Todorov distingue dos diferentes; Genette se inspira en la terminología de Brooks y Warren para llegar al término "focalización"; Bal reconoce que el concepto no es nuevo y, de alguna manera, retoma la idea de la cámara de Friedman. Entonces, si hay un lazo que une todas las tentativas por llegar al establecimiento del concepto de focalización, en todas puede detectarse la misma preocupación por estudiar la relación entre narrador/lector/mundo narrado y las maneras de elaborar el mundo de la ficción.

Ahora bien, si ha habido en realidad una evolución desde el concepto de "punto de vista" hasta el de "focalización", ¿ ¿significa esto que el primer término debe descartarse definitivamente? Creo que no. Más que abandono del término -y tal vez del conceptode punto de vista, debe hablarse de un desplazamiento hacia el interior del texto -desplazamiento que ha quedado ilustrado en los apartados 1 y 2 de este trabajo-, para el que se ha adoptado el término focalización. ${ }^{14}$

Es importante recordar dos cosas: 1) para Greimas (1982), el concepto de focalización "no es sino provisional: no da cuenta de todos los modos de presencia del observador [...]; no explica tampoco la constitución de los espacios cognoscitivos parciales, caracterizados por la presencia -dentro de los programas pragmáticos- de dos sujetos cognoscitivos en comunicación” (p. 179); ${ }^{15}$ 2) hay que

${ }^{14}$ Podría decirse que, para los estudiosos de este problema, lo que antes se llamaba punto de vista, ahora se llama focalización. Greimas (1982) escribe: "la antigua noción de punto de vista" y M. Bal (1985): "nombre nuevo para un concepto parcialmente antiguo".

15 Sin embargo, el problema del punto de vista no dejó de llamar la atención de Greimas. En Greimas (1975), definió el punto de vista como "la identificación del sujeto discursivo con uno u otro de los sujetos narrativos" (p. 171). Así, afirma que el problema de "¿quién ve?" queda resuelto en el cuento "Deux amis" porque Maupassant no sólo no describe a los soldados prusianos, sino que estos son "vistos" por su delegado S1 -Sau- 
distinguir tres valores diferentes: ${ }^{16}$ a) focalización, como "presentación"; b) perspectiva, como la distancia del focalizador con respecto a lo focalizado; c) punto de vista que involucra una interpretación.

Si esto es así, el concepto de punto de vista, tal como surge a fines del siglo XIX, con las preocupaciones de James, ha sufrido una doble modificación. Primero, el desplazamiento para designar algo que existe sólo en el interior del texto, con la consiguiente adopción del término focalización; segundo, utilizado para designar lo que involucra la interpretación, conserva su sentido narrador/historia, pero ya sin la confusión con respecto al autor.

En fin, el desarrollo del concepto de focalización -que es actualmente un campo abierto, donde la narratología puede probar sus fuerzas- es una muestra de cómo surge y se desarrolla un concepto teórico y de cómo un instrumento de análisis es discutido, reformulado, perfeccionado y sometido a prueba en esa labor apasionante que es el análisis del texto.

\section{Bibliografía}

BAL, M. (1985). Teoría de la narrativa. Madrid. Cátedra.

Benveniste, É. (1966). Problèmes de linguistique générale. Paris. Gallimard.

BuIn, G. (1954). Stendhal et les problèmes du roman. Paris. Joseph Corti. Воотн, W. C. (1961). The rethoric of fiction. Chicago. The University of Chicago Press.

Bourneuf, R. y Ouellet, R. (1975). La novela. Barcelona. Ariel.

vage y Morissot, los dos amigos. Además, la descripción del oficial prusiano es la figura hipotáctica del Mont-Valérien y forma parte, "como una lectura particular, de lo visto, del universo cognoscitivo de los dos amigos" (p. 172).

16 Según se discutió en una de las sesiones del Seminario de narratología -enero de 1986- de la Maestría en Ciencias del Lenguaje de la Universidad Autónoma de Puebla. El seminario fue dirigido por el Dr. Renato Prada. 
Brooks, C. \& Warren, R. P. (1947). Understanding fiction. Nueva York. Crofts \& Co.

Ducrot, O. y Todorov, T. (1972). Diccionario enciclopédico de las ciencias del lenguaje. México. Siglo xxi Editores.

Forster, E. M. (1927). Aspects of the novel. Nueva York. Harcourt, Brace \& World.

Friedman, Norman. (1955). Point of view in fiction: the development of a critical concept. PMLA, 70(5), pp. 1160-1184. Columbia University.

Genette, G. (1966). Figures I. Paris. Seuil.

Genette, G. (1969). Figures II. Paris. Seuil.

Genette, G. (1972). Figures III. Paris. Seuil.

Greimas, A. J. y Courtés J. (1982). Semiótica. Diccionario razonado de la teoría del lenguaje. Madrid. Gredos.

Greimas, A. J. (1975). Maupassant. La sémiotique du texte: exercices pratiques. Paris. Seuil.

James, H. (1934). The art of the novel. Critical prefaces. Nueva York.

LubBock, P. (1921). The craft of fiction. Nueva York. The Viking Press.

Magny, C. (1946). L'age du roman américain. Paris. Seuil.

Patillon, M. (1974). Précis d'analyse littéraire. Paris. Nathan Université.

Poulllon, J. (1970). Tiempo y novela. Buenos Aires. Ed. Paidós.

Prévost, J. (1942). La création chez Stendhal. Marseille. French \& European Publications.

Rubio de LérTora, P. (1981). El concepto de focalización. Su aplicación a la cuentística de Onelio Jorge Cardoso. Semiosis, (6). Universidad Veracruzana.

Sartre, J. P. (1947). Situations I. Paris. Gallimard.

Stendhal, G. (1840). La Chartreuse de Parme. Paris. Ambroise Dupont, Éditor.

Todorov, T. (1967). Littérature et signification. Paris. Larousse.

Todorov, T. (1971). ¿Qué es el estructuralismo? Buenos Aires. Losada.

Tolstoi, L. (1878). Ana Karenina, vir. Paris. Poche.

Uspensky, B. (1970). Poétique de la composition. Paris. Seuil. 
Valette, B. (1985). Esthétique du roman moderne. Paris. Nathan Université.

Zéraffa, M. (1971). Personne et personnage. Paris. Klincksieck. 